\title{
Emotion Detection using Facial Expressions with Convolution Neural Networks
}

\author{
D Aruna Kumari, M N L Anuradha
}

\begin{abstract}
Artificial intelligence systems to perceive human feeling have pulled in much research premium, and potential uses of such frameworks flourish, spreading over areas, for example, client mindful showcasing, health monitoring wellbeing observing, and genuinely shrewd robotic interfaces. Human are enthusiastic creatures and it assumes a significant job behind their thoughts and activity. In this way, it is important that emotion handling capacities are assimilated for planning of human condition. The investigation, recognition and synthesis of feelings can plan the human environment. In this procedure the data uses, for example, sound, visual, composed and mental data. An epic research theme to be developed in the Human Computer Interaction field is Emotion Recognition utilizing Facial Expressions.
\end{abstract}

Keywords : face detection, speech detection, neural networks

\section{INTRODUCTION}

Because of the regularly augmenting use and request of PC the requirement for an increasingly common correspondence interface among human and PC had normally gone to the power. To accomplish this point, a PC would need to be reactions diversely in current circumstance dependent on the recognition which is seen by the $\mathrm{PC}$. To make the communication among human and PC all the more normally, it is significant for the last can react to the feelings of people similarly like human in its position will do. To accomplish the objective that PC can recognize the feeling by outward appearance. There are some essential emotions given by various researchers are happiness, sadness, anger, disgust, surprise and fear. Fundamentally, Emotion Recognition manages the investigation of deducing feelings and techniques utilized for inference. Emotions can be perceived from face expressions and voice signals.

Different strategies have been created to discover the feelings, for example, flag handling, AI, neural systems, PC vision ,signal processing, machine learning, and neural networks. Analyzing and recognizing the emotions are being studied and some are under development stage all over the world. Generally facial expressions can classified into 6 Happy, Sad, Anger, Disgust, Surprise and Fear[1].

This work plans a smart system. This brilliant enthusiastic acknowledgment framework reacts to Facial articulations, at whatever point it identified the outward appearances it comprehend the feelings and produces the result. It likewise tells the best way to extricates face land marks from face and afterward changes over to features .Then we will prepare this

Revised Version Manuscript Received on 16 September, 2019.

Dr.D Aruna Kumari at Department of CSE, VJIT, Hyderabad, Telangana , arunakumari@vjit.ac.in

M N L Anuradha ${ }^{2}$, Associate Professor in Department of Mathematics at Vidya Jyothi Institute of Technology, Hyderabad, anuradha.chennuru@gmail.com with CNN for recognizing the emotions. One of the AI methods utilized is CNN[10].

\section{REVIEW CRITERIA}

This journal uses double-blind review process, which means that both the reviewer (s) and author (s) identities concealed from the reviewers, and vice versa, throughout the review process. All submitted manuscripts are reviewed by three reviewer one from India and rest two from overseas. There should be proper comments of the reviewers for the purpose of acceptance/ rejection. There should be minimum 01 to 02 week time window for it. Convolution Neural Networks, and all the more explicitly convolution layers in neural systems, give a ground-breaking approach to remove highlights from pictures. When the system has separated the significant features from a picture, you can utilize in the manner you need[11].

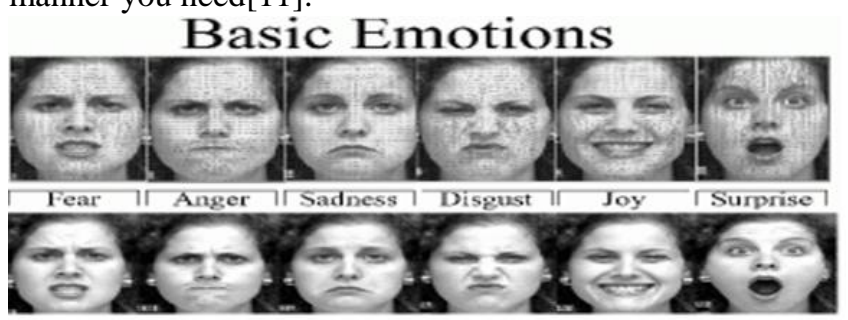

2.Related Work Yu and Zhang worked and achieved state-of-the-art results in EmotiW using CNNs to perform Facial expression recogntion in 2015. They worked with CNNs with five convolution layers .Specifically, Yu and Zhang applied transformations to the input images at train time. At test time, their model generated predictions for multiple perturbations of each test example and voted on the class label to produce a final answer. Also attractive is that they introduced stochastic pooling instead of using max pooling because of its better efficiency on training data. Kim et al. achieved a test accuracy. $\mathrm{Xu}$ [2] has proposed a model with Deep CNN for Visual Sentiment identification they worked on Convolution Neural Networks which were pre trained on Object recognition data to perform sentiment analysis on images collected from Twitter and Tumblr. [3] You, Quanzeng has worked on sentiment analysis of Robust image by prepared deep networks [3] uses VGGImageNet for image detection. Karayev [4] has performed based on space ana saliency features. Dellagiacoma[6]has worked on natural images and they performed emotion classification with bag of emotions method.[5] they have worked on Speech recognition through gender. Emotion based classification of natural images by Dellagiacoma et al. [5] uses MPEG7 color and edge descriptors for executing sentiment classification and 
compares the results with Bag of Emotions

\section{Methodology}

Detecting the Emotions from images have several applications, like labeling public media images such as usability studies, focus groups, security purpose, sales and training, or healthcare.

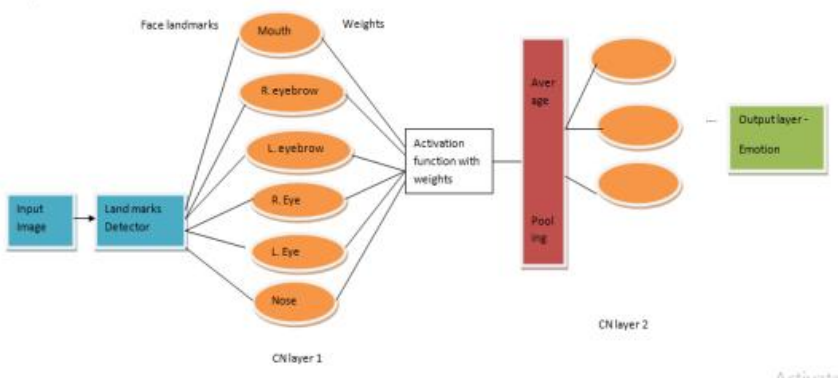

Face Detection:

Fig 2: Approach

Emotion analysis from Facial expression is a recent research area because of its prospective applications in the many areas such as human being-robotic interaction, human-machine interfaces, safe driving, and health-care. In spite of the noteworthy upgrades, facial expression recognition is yet a difficult issue that sit tight for an ever increasing number of exact calculations. This article displays another model that is equipped for perceiving emotion detection from facial expressions by utilizing profound Convolution Neural Network (CNN)

\section{Algorithm}

Our algorithm takes the following facial regions as face land marks.

a. Mouth (lips)

b. Right eyebrow

c. Left eyebrow

d. Right eye

e. Left eye

f. Nose

Step1: Retrieve picture from a web camera

Step2: find out Face Landmarks

Step3: prepare a machine learning algorithm (we will use C.N.N)

Step4: Discover the emotions

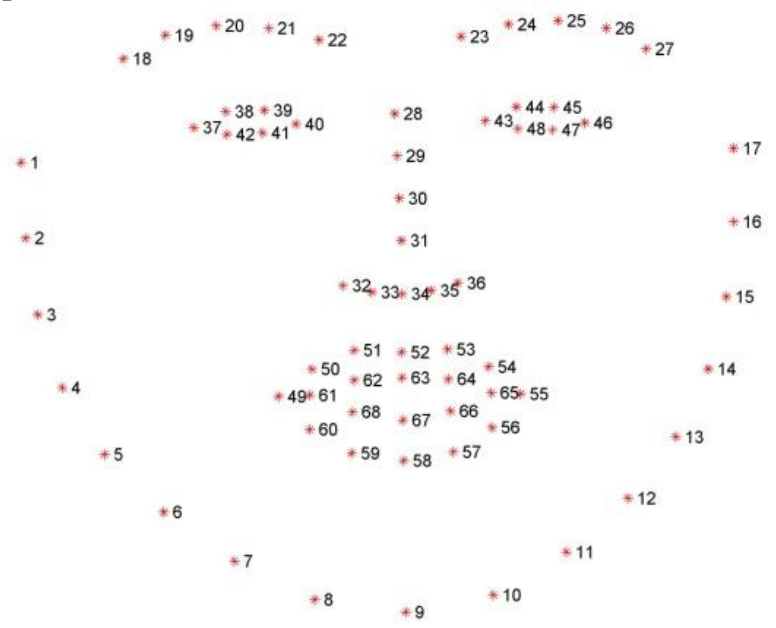

Fig 3: Template for Face land marks
These explanations are a piece of the 68 point iBUG $300-\mathrm{W}$ dataset which the dlib facial milestone indicator was prepared on.

It's imperative to take note of that different kinds of facial milestone locators exist, including the 194 point model that can be prepared on the HELEN dataset.

Despite which dataset is utilized, the equivalent dlib system can be utilized to prepare a shape indicator on the information preparing information - this is helpful on the off chance that you might want to prepare facial milestone identifiers or custom shape indicators of your own.

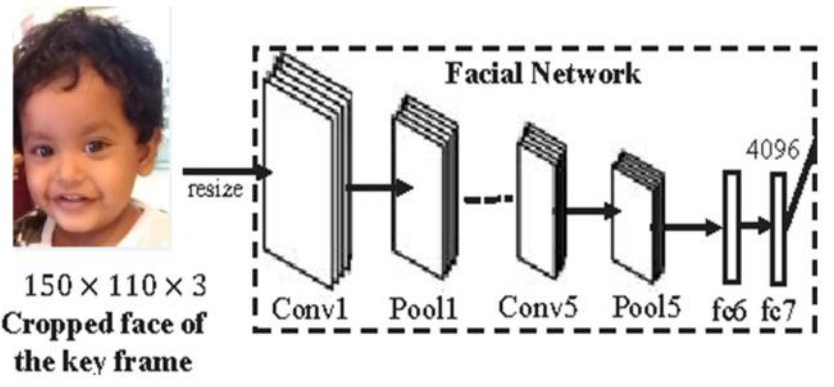

Fig 4: Convolution Neural networks

\section{a). facial landmarks with dlib, OpenCV, and Python}

The utility method to be used is rect_to_bb , short for "rectangle to bounding box":

def rect_to_bb(rect):

$\mathrm{x}=$ rect.left ()$\quad / /$ left position

$\mathrm{y}=$ rect.top ()$\quad / /$ top coordinates

$\mathrm{w}=$ rect.right ()$-\mathrm{x} / /$ right points

$\mathrm{h}=$ rect.bottom ()$-\mathrm{y} / /$ bottom points

return $(\mathrm{x}, \mathrm{y}, \mathrm{w}, \mathrm{h})$

we can also use the method called shape_to_np

one of the detector is dlib which returns a shape object having $68(x, y)$-coordinates of the landmark regions of the face. By using two functions we can detect the facial landmarks from images.

Usually This will generate your face with a group of dots from which emotions can be traced.

\section{b) Extracting features from the faces} After the land marks are detected

The principal activity is discover approaches to change these pleasant spots overlaid all over into features to bolster the classifier. features are little bits of data that portray the item or article express that we are endeavoring to isolate into classes. The dat file can be found with groups of dots[8] With the help of face landmarks coordinates, emotions will be identified. Here we derive the coordinates of landmarks. coordinates are the main sources of information about features, and this might be useful and you can also try to derive other sources that will tell the classifier more about what is happening on the face.

To begin, take a gander at the coordinates. They may change as my face moves to various pieces of the casing. I could be communicating a similar feeling in the upper left of a 
picture as in the base right of another picture, however the subsequent organize grid would express extraordinary numerical extents. Be that as it may, the connections between the coordinates will be comparative in the two lattices so some data is available in an area invariant structure.

Perhaps the most direct approach to expel numerical contrasts beginning from appearances in better spaces of the portrait would regulate the coordinates. somewhere in the range of 0 and 1 . This is easily done by

$$
X_{\text {norm }}=\frac{X-X_{\min }}{X_{\max }-X_{\min }}
$$

xnormal $=$

$[(\mathbf{j}-\min (x \operatorname{list} 1)) /(\max (x \operatorname{list} 1)-\min (x \operatorname{list} 1))$ for $\mathbf{j}$ in xlist1]

ynormal $=[\mathrm{j}-\min (\mathrm{ylist} 2)) /(\max (\mathrm{ylist} 2)-\min (\operatorname{ylist} 2))$ for $\mathrm{j}$ in ylist2]

\section{Experimental Results}

We have trained the data sets and tested in python dlib and openCv, we have reached almost $87 \%$ of accuracy with respective prediction of the emotion
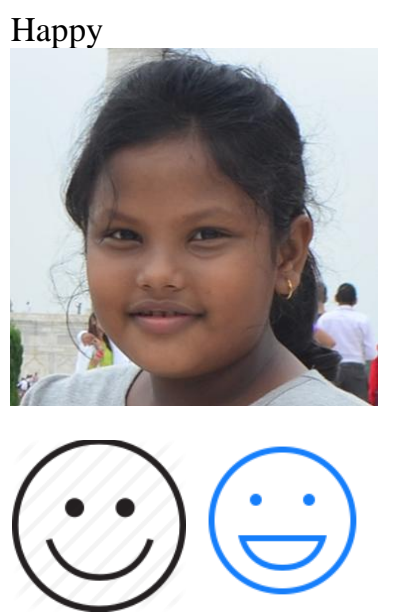

Surprise

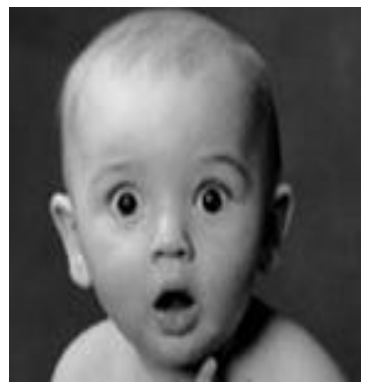

Surprise- icon

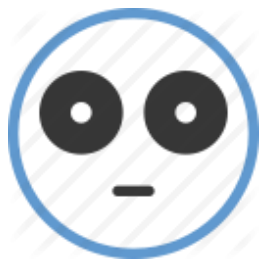

fear: 0.04916767

neutral: 0.00806644

happy: 0.13546762

sadness: 0.03670473

surprise: 0.31565646

anger: 0.01434529

contempt: 0.15626157

disgust: 0.01217962

fear: 0.00434321

surprise: 0.378776

happy: 0.00522828

sadness: 0.07478257

neutral: 0.35112345
Sad

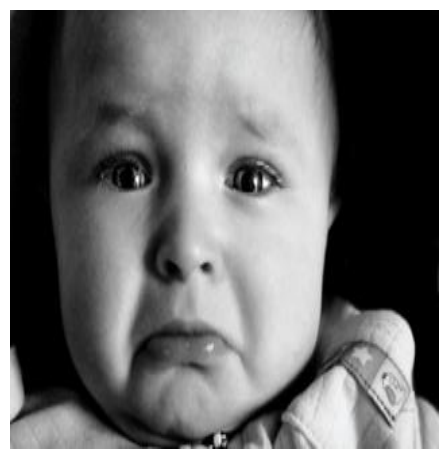

anger: 0.037256873

contempt: 0.05605531

disgust: 0.13482577

fear: 0.16447784

neutral: 0.09523987

happy: 0.20552734

sadness: 0.21521671

surprise: 0.018256147

\section{Sad - icons}
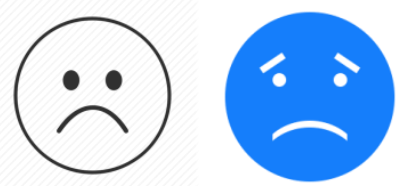

it is very close to Happy, every so often land marks may generates ambigutiy among the emotions because of the gap between land marks.

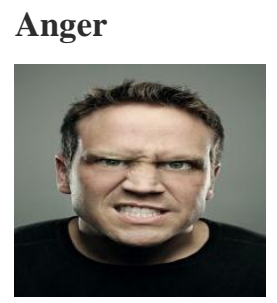

anger: 0.3226787

contempt: 0.24435082

disgust: 0.06127896

fear: 0.02427509

neutral: 0.20176093

Anger- icon

happy: 0.03169800

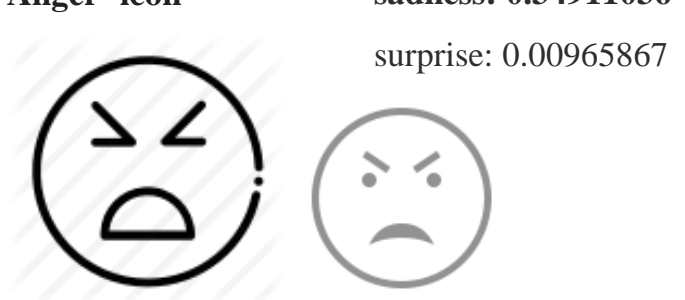

\section{CONCLUSIONS}

In this work, the task of facial expression recognition is implemented in Python, dlib and openCv. It has extracted the land marks with dlib. Input images have been then identified with features and respective emotions have been identified. In the experimental results, we got the accurate results with some limitations. The approach for finding emotions from features is based Euclidian distance. For some emotions it is giving near values even though they are not of that emotion.

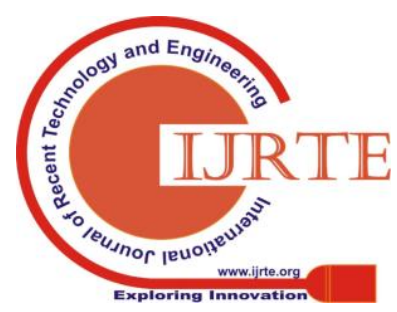




\section{REFERENCES}

[1] C. M. Lee, S. Yildirim, M. Bulut, A. Kazemzadeh, C. Busso, Z. Deng, S. Lee, \& S. Narayanan, Emotion recognition based on phoneme classes, Proc. ICSLP, Jeju, Korea, 2004, 889-892.

[2] Xu, Can, et al. "Visual sentiment prediction with deep convolutional neural networks." arXiv preprint arXiv:1411.5731 (2014).

[3] You, Quanzeng, et al. "Robust image sentiment analysis using progressively trained and domain transferred deep networks." arXiv preprint arXiv:1509.06041 (2015).

[4] Karayev, Sergey, et al. "Recognizing image style." arXiv preprint arXiv:1311.3715 (2013).

[5] T. Vogt \& E. André, Improving Automatic Emotion Recognition from Speech via Gender Differentiation, Proc. Language Resources and Evaluation Conference, Genoa, Italy, 2006, 1123-1126.

[6] Dellagiacoma, Michela, et al. "Emotion based classification of natural images." Proceedings of the 2011 international workshop on DETecting and Exploiting Cultural diversiTy on the social web. ACM, 2011.

[7] D. Jiang \& L. Cai, Speech Emotion Classification with the Combination of Statistic Features and Temporal Features, Proc. IEEE International Conference on multimedia, Taipei , Taiwan, China, 2004, 1967-1970.

[8] Prudhvi Raj Dachapally" Facial Emotion Detection Using Convolutional Neural Networks and Representational Autoencoder Units"

[9] Gary B. Huang et al. "Labeled Faces in the Wild: A Database for Studying Face Recognition in Unconstrained Environments." University of Massachusetts, Amherst, Technical Report 07-49, October, 2007.

[10] Francois Chollet, Keras, GitHub, https://github.com/fchollet/keras, 2015.

[11] Alex Krizhevsky et al. "ImageNet Classification with Deep Convolutional Neural Networks", Neural Information Processing Systems (NIPS), 2012.

\section{AUTHORS PROFILE}

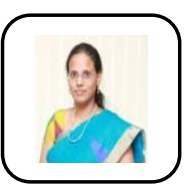

Dr Aruna Kumari Professor at Department of CSE,VJIT, Hyderabad, Telangana India. She is Fellow of CSI( FCSI), and Fellow of IEEE (FIEEE). She is DST Young Scientist Awardee (Govt. of India). She is Ph.D Supervisor at Department of CSE KL-University, Guntur, Andhra Pradesh. Data mining, Cloud Computing, Privacy \& Security of Big Data, Big Data Analytics are her interested areas of research. She has more than 70 research articles in International Journals and Conferences. She is Currently working on research project "Design and Development of Effective Privacy Preserving Data Mining for Cardiac Cancer and Diabetic Health Care" funded by DST-SERB. She has organized several National and International Conferences and Workshops.

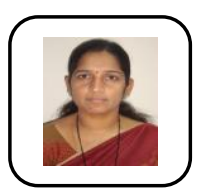

Ms.M.N.L.Anuradha, Received Post graduate Degree in Mathematics from Osmania University, M.Phil (Mathematics ) from Madurai Kamaraj University and MTech (Computer Science \& Technology) from JNTUH and currently pursuing $\mathrm{Ph} . \mathrm{D}$ (Mathematics) from KL University. She is having19 years of Teaching Experience and currently working as an Associate Professor in Department of Mathematics at Vidya Jyothi Institute of Technology, Hyderabad. She has published nearly10 publications in the areas of Database, Data Mining, Cloud Computing and Network Security. She is associated with various Professional bodies like ISTE, IA Engg, IACSIT, and CSTA. 